\title{
Calculation of Head Sensitivity Function from 3-D Magnetic Fields
}

\author{
Edward R. Burke \\ Laboratory for Physical Sciences, College Park, MD 20740
}

Romel D. Gomez, Rajiv Madabhushi, and Isaak D. Mayergoyz

Department of Electrical Engineering, Institute for Advanced Computer Studies, University of Maryland, College Park, MD 20742

\begin{abstract}
A head sensitivity function is calculated using 3-D magnetic fields. The 3-D magnetic fields are calculated for recorded tracks with finite widths. The magnetization in the medium is expressed in 2-D Fourier series in the plane of the film and is assumed uniform through the thickness of the film. The reproduce head is idealized. These techniques are applied to data found in the literature for very narrow tracks $(\sim 0.5 \mu \mathrm{m})$. A surprising amount of the experimental data can be explained by the finite width of the tracks. The fine structure of the measurements can then be isolated and attributed to the particular features of the individual heads.
\end{abstract}

\section{INTRODUCTION}

The terms "head sensitivity function", "head response function", and "head field sensitivity function" are all used interchangeably in the literature. The reason for this is that if one calculates the response of a head by using the reciprocity theorem, then there are numerous instances of recorded magnetizations where the response waveform is the same as the head field waveform [1]. In this paper we will retain the terminology "head sensitivity function" but what we are calculating is the response of the head not only as it moves along the track but also as it is moved across the track. We do not invoke reciprocity but instead calculate the voltage from the time rate of change of the flux through an idealized head. The flux is obtained by integrating the field emanating from a finite width track.

There are numerous experimental techniques to determine a head sensitivity function from recording measurements [2], (and references therein). A common feature of these techniques is the desire to obtain information about the readback response across the width of the track. In a previous paper [3] we showed how to calculate the magnetic fields from finite width tracks. The readback from an idealized head could then be determined. In this paper we apply those techniques to measurements found in the literature. By comparing the calculations to the measured values, one can then determine which effects are due to the variations in the fields and which are due to the particular features of the experimental heads.

\section{A. Read back Voltage}

In [3] we showed how the magnetic fields emanating from a recorded surface containing an infinite number of identical tracks could be calculated. The solutions were obtained by

Manuscript received April 4, 1994. assuming that the magnetization is uniform through the thickness of the film and can be represented by Fourier series in the other two dimensions. These fields were then used to calculate the readback voltage from an idealized head with the result, for a track in the $x$ direction, given by

$$
\begin{aligned}
V= & \frac{\mu}{\mu+1} 4 \pi M_{s} v N W_{h_{n, m}} X_{n} Y_{m}\left(k_{x} / k\right)^{3} \frac{\sin k_{y} W_{h} / 2}{k_{y} W_{h} / 2} \times \\
& e^{-k z}\left(1-e^{-k \delta}\right) \cos k_{x} x \cos k_{y} y,
\end{aligned}
$$

where $\mu$ is the magnetic permeability of the head, $M_{s}$ is the saturation magnetization of the recording medium, $v$ is the velocity of the head relative to the medium, $N$ is the number of head turns, $W_{h}$ is the width of the head, $k_{x}$ and $k_{y}$ are the wave numbers in the $x$ and $y$ directions $\left(k_{x}=2 \pi n / \lambda_{\mathrm{x}}\right.$, $k_{y}=2 \pi \mathrm{m} / \lambda_{\mathrm{y}}$ ) where $\lambda_{\mathrm{x}}$ and $\lambda_{\mathrm{y}}$ are the wavelengths, $X_{n}$ and $Y_{m}$ are the Fourier coefficients for the magnetization distribution in the $x$ and $y$ directions, $\delta$ is the thickness of the recorded medium and $k=\sqrt{k_{x}^{2}+k_{y}^{2}}$. The point $(x, y, z)$ is the location of the center of the head, where $z$ is the distance from the medium to the head. For a head with a gap $g$, each term in (1) will be multiplied by the factor $\sin \left(k_{x} g / 2\right) /\left(k_{x} g / 2\right)$, which can be obtained by spreading the $N$ turns over a distance $g$ and then averaging $H_{x}$ over this distance when calculating the flux through the head.

Although (1) was derived for an idealized head, it is more general than what would be derived using a Karlquist head [4] and the reciprocity theorem. If the 2-D Fourier series that we use for the magnetization is used in a generalized reciprocity integral, the sampling factor for the head width is obtained, as in (1). On the other hand, using a Karlquist head field that is not a function of $y$ will have the effect of reducing $k$ in (1) to $k_{x}$ and much of the 3-D structure will be lost.

\section{B. Fourier Distributions}

For (1) to be useful, we must find Fourier series that are both representative of the magnetization pattern being studied and easy to use. To this end, we have made an extensive study of numerous Fourier series and have found that the most useful ones are series with arctan transitions. The Fourier series that we use can be shown to be identical to the series that would be constructed from the linear superposition principle [5]. The Fourier coefficients that correspond to a symmetric, repetitive magnetization pattern in the $x$ direction are given by [3] 


$$
x_{n}=4 e^{-2 \pi(2 n+1) a_{x} / \lambda} x / \pi(2 n+1),
$$

where $a_{x}$ is the transition length.

The coefficients that represent the magnetization for identical tracks in the track width direction, $y$, are given by [3]

$Y_{m}=\frac{2 e^{-\frac{2 \pi m a}{W+b}}}{\pi m} \sin \frac{\pi m W}{W+b}, m>0 ; Y_{m}=\frac{W}{W+b}, m=0_{(3)}$ where $W$ is the track width, $b$ is the track separation, and $a_{y}$ is the arctan transition length in the $y$ direction. Using (1)-(3), we can now calculate the response for an idealized head. The pickup voltage can be calculated as a function of the position of the head relative to the tracks, the track widths, the track separations, and the transition lengths of the recorded signals. The task now is to apply these calculations to experimental data.

\section{EXPERIMENTAL DATA}

The first data we will consider was contained in a paper by Lambert, Sargent and Williams [2] in which the head sensitivity function was determined by writing a very narrow track $(\sim 0.5 \mu)$ by dc-erasing the sides of a conventional track. The readback voltage from isolated pulses was then measured along the track for different positions across the track. The amplitude data could then be plotted as a surface plot for both the distances along and across the track.

At first glance it might seem inappropriate to apply our techniques to this experiment since the theory is for repetitive data on identical tracks while the experiment is for isolated transitions on a single track. However, this can be regarded as a sort of generalization that can be simply achieved by moving the tracks and the transitions far apart so that they are effectively isolated. This means that more terms must be included in the Fourier series, but we have found that this presents no great problems even when the calculations are performed on a PC.

\section{A. Magnetization}

An example of the technique is shown in Fig. 1. In Fig. 1(a) we have plotted the magnetization distribution for a single transition on an isolated track given by

$M_{x}=\frac{2}{\pi^{2}} \tan ^{-1} \frac{x}{a_{x}}\left[\tan ^{-1} \frac{y+W / 2}{a_{y}}-\tan ^{-1} \frac{y-W / 2}{a_{y}}\right]$,(4)

where $W$ is the width of the track and $a_{x}, a_{y}$ are the transition lengths in the $x, y$ directions. In Fig. 1(b) we have plotted the Fourier transforms representation corresponding to (2)-(3) for the same values as for Fig.1(a), ( $W=0.525, a_{x}=0.68$, $a_{y}=0.09$ ). In Fig. 1(b) we chose $\lambda_{x}=40$ for the wavelength, and $b=25$ for the track separation. With these large values, relative to the track width and the transition lengths, the Fourier series representation is almost identical to the exact expression, as can be seen from Figs. 1(a) and 1(b). The fine structure in Fig.
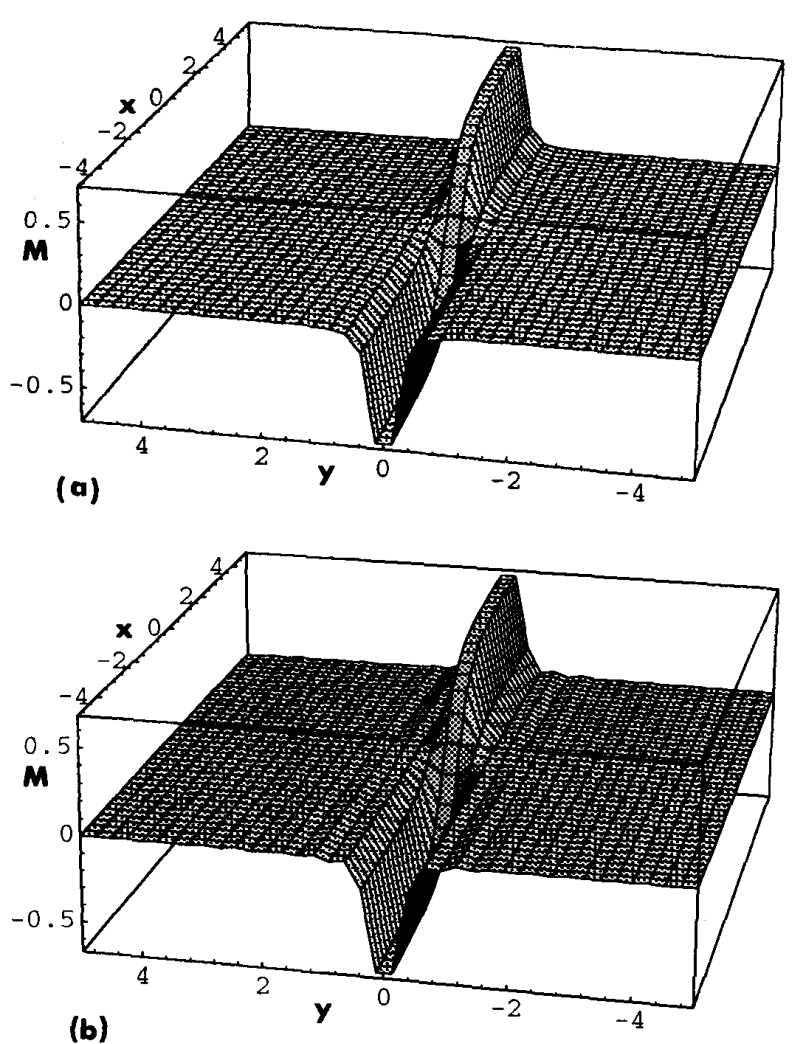

Fig. 1. (a) Magnetization of a transition on a single track given by (4). (b) Fourier series representation of the magnetization given by (2)-(3). (Rear views for visual clarity)

1(b) is the result of using only a finite number of terms $(n=16, m=40)$. Fewer terms are needed when calculating the voltage because of the attenuation of the higher harmonics.

\section{B. Head Response}

The head response may now be calculated using (1)-(3) and is shown in Fig. 2. The values used for the calculations were: $z=0.17 \mu \mathrm{m}, \delta=0.06 \mu \mathrm{m}$, which are values from the experiments; $W_{h}=14 \mu \mathrm{m}, W=0.525 \mu \mathrm{m}, a_{x}=0.68 \mu \mathrm{m}, a_{y}=0.09$ $\mu \mathrm{m}$, which were chosen by trial and error to give the best fit to the data; and $\lambda_{x}=40 \mu \mathrm{m}, b=25 \mu \mathrm{m}$, which were chosen to isolate the pulse and the track as explained above.

The best-fit values are quite reasonable. The experimental head had pole tips with widths of $12 \mu \mathrm{m}$ and $15 \mu \mathrm{m}$, so that the $14 \mu \mathrm{m}$ value for $W_{h}$ that we have determined can be thought of as the effective read width of the head. In [2], the microtrack was inferred to be $0.5 \mu \mathrm{m}$ from the observed amplitude compared to the amplitude for the originally assumed $12 \mu \mathrm{m}$ wide track. Our calculations, however, show that the amplitude is not linear with the track width for small track widths. The trackwidth value of $0.525 \mu \mathrm{m}$ that fits the data also gives an amplitude that is $1 / 24$ the amplitude of a 14 
$\mu \mathrm{m}$ trackwidth, which would be consistent with the amplitude measurements in [2]. It would be interesting to measure these widths more accurately, such as by imaging techniques, to resolve these minor differences.

The best-fit value for the transition length along the track, $a_{X}=0.68 \mu \mathrm{m}$, differs from that found in [2], $\left(a_{x}=0.19 \mu \mathrm{m}\right)$. This can be attributed to the difference in the approach applied to determine this value. In [2], a calculation based on the writing process using the Williams-Comstock model [6] was used. The transition lengths obtained by this method can be thought of as the minimum value achieved in the write process for a given set of magnetic parameters. The best-fit value that we obtain is based on the transition length as written. The transition length can also be determined by imaging techniques [7], and again, it would be interesting to perform the experiments to resolve any differences.

The best-fit value for the transition length perpendicular to the track, $a_{y}=0.09 \mu \mathrm{m}$, is reasonable since the transition can be much sharper in this direction because there are no free magnetic charges at the track edges (the magnetization is parallel to the transition). It should be mentioned that the magnetization shown in Fig. 1 is not exactly the same as in the experiments since in the experiments the track edges were formed by dc-erasing. The magnetization off-track would then be either $+\mathrm{M}_{s}$ or $-\mathrm{M}_{s}$, rather than zero as shown in Fig. 1 . Adding or subtracting a constant magnetization would, however, have no effect on the calculated magnetic fields.

The most surprising feature of the calculations shown in Fig. 2 are the "shoulders" on the sides of the response and the undershoot along the track. They appear almost exactly as seen in the experimental results. This structure was attributed in [2] to the finite dimensions of the poletips. In our calculations for an idealized head, the poletips are assumed infinite and the structure seen in Fig. 2 is caused by the variations in the magnetic fields across the width of the track. Equation (1) is thus seen to be an extremely useful description for the response of a pickup head for finite width tracks.

\section{Other Experiments}

We have applied (1) to other experimental data found in the literature, and although space precludes a full discussion of these studies, we should mention the cases where (1) cannot give a complete description of the results. Since (1) was derived for an idealized head with an infinite extent it will not describe effects due to finite pole tips. Examples are given in a paper by Lambert and Williams [8] in which the spectral response for a discrete track was measured for various locations across the track and as a function of the density of recording. Measuring the spectral response as a function of data density would be equivalent to taking only the first harmonic in (1), $n$ $=0$, and converting the wavelength in the $\mathrm{x}$ direction to a density of recording. While our techniques could fit the data to which it was applied in a broad sense, it could not fit the fine structure in the data presumably caused by the finite pole tips of the heads. Lambert and Williams also managed to find such structures when they measured the effective readback width of their head as a function of data density. We found that the effective width increased for low densities and leveled off for high densities, the same as found by Lambert and Williams, but again, we could not reproduce the fine structure.

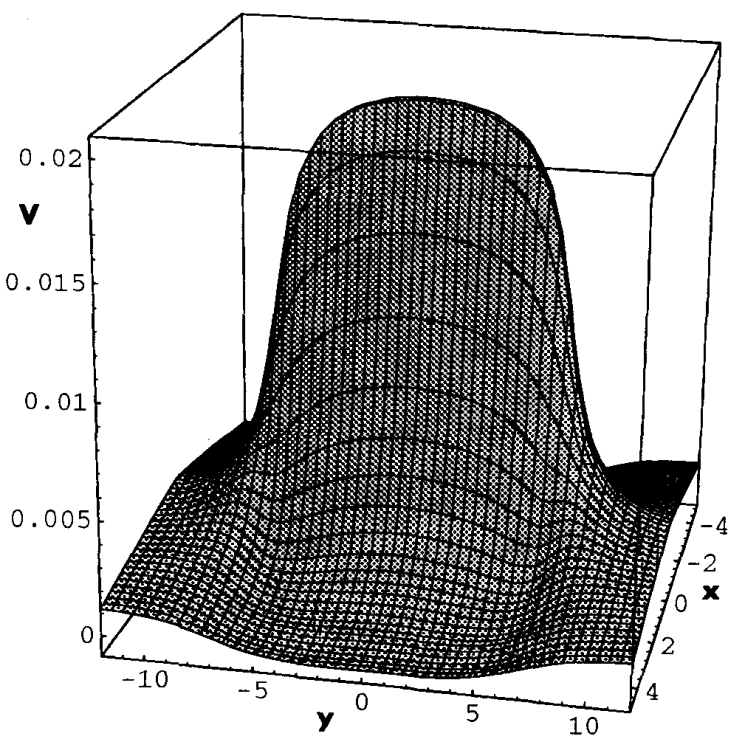

Fig. 2. Read back amplitude acquired from (1)-(3).

\section{ACKNOWLEDGMENT}

The authors wish to thank Shannon Keehn for invaluable assistance with the computer calculations.

\section{REFERENCES}

[1] B. K. Middleton, "Recording and reproducing processes," in Magnetic Recording, vol. I: Technology, C. D. Mee and E. D. Daniel, Eds. New York: McGraw-Hill, 1987, pp. 22-97.

[2] S. E. Lambert, F. D. Sargent, and M. L. Williams, "Head sensitivity function determined from recording measurements," IEEE Trans on Magn., vol. 27, no. 6, pp. 4458-4460, 1991.

[3] E. R. Burke, R. D. Gomez, R. Madabhushi, and I. D. Mayergoyz, "Computation of magnetic fields from recording surfaces with multiple tracks," J. Appl. Phys., tvol. 75, no. 10, pp. 5759-5761, 1994.

[4] O. Karlquist, "Calculation of the magnetic field in the ferromagnetic layer of a magnetic drum," Trans. Roy. Inst. Technol. Stockholm, vol. 86, pp. 1-27, 1954

[5] J. C. Mallinson, "Applications of fourier transforms in digital magnetic recording theory," IEEE Trans. on Magn., vol. 10, no. 1, pp 69-77, 1974.

[6] M. L. Williams and R. L. Comstock, "An analytical model of the write process in digital magnetic recording," A. I. P. Conf. Proc., vol. 5, 1972, p. 738 .

[7] R. D. Gomez, E. R. Burke, A. A. Adly, I. D. Mayergoyz, J. A. Gorezyca, and M. H. Kryder, 'Magnetic force scanning tunneling $6180-6182,1993$

[8] S. E. Lambert, and M. L. Williams, "Recording head characterization using $1 \mu \mathrm{m}$ wide discrete tracks," IEEE Trans on Magn., vol. 24 , no. 6. pp. 2832-2834, 1988 . 